\title{
HIV Surveillance: A Global Perspective
}

\author{
*Elizabeth Pisani, †Stefano Lazzari, *Neff Walker, and *Bernhard Schwartländer \\ *The Joint United Nations Program on HIV/AIDS; †The World Health Organization, Geneva, Switzerland
}

\begin{abstract}
Summary: This article provides an overview of recommendations for HIV surveillance. Results of surveillance are used in practice to inform program decisions, judge the effectiveness of the national response, lobby for effective programs, and to provide accurate measures of trends and the absolute state of the epidemic. Recommended surveillance activities differ for different epidemic situations-epidemics that are concentrated in defined groups with high-risk behavior, and epidemics that are well established among heterosexuals in the population at large. Surveillance systems in countries with different levels of the epidemic face major challenges, most of which revolve around identifying and obtaining information from representative samples of the at-risk populations. A brief examination of surveillance systems in Botswana and Vietnam illustrate how these challenges are being met in practice. While there is room for improvement, HIV surveillance systems in many developing countries are relatively robust, and are growing stronger all the time. In most countries, however, insufficient use is made of the information generated by these systems in terms of strengthening HIV prevention and care programs. Key Words: HIV-AIDSSentinel surveillance.
\end{abstract}

Throughout the 1980s, HIV and AIDS were discovered in one population after another around the world, and a great deal of energy was focused on setting up systems to track the spread of the virus. In the late 1990s, a number of countries worked with international organizations to distill the lessons learned to date, and to strengthen and improve existing surveillance systems. The result has been a framework-termed "second generation HIV surveillance"- that aims to tailor surveillance systems to the needs of specific epidemic states (1). Second generation systems use behavioral as well as biological data to increase the explanatory power of surveillance systems in both predicting and tracking the course of the HIV epidemic in a given country.

Second generation HIV surveillance was developed to meet HIV surveillance needs identified by many coun-

Elizabeth Pisani is now at Family Health International in Jakarta, Indonesia, and Bernhard Schwartländer is at the World Health Organization.

Address correspondence and reprint requests to Bernhard Schwartländer, WHO, 20 Avenue Appia, CH-1211, Geneva 27 Switzerland; e-mail: schwartlanderb@who.int tries. In the early years of the epidemic, countries were encouraged to set up surveillance systems which had as a primary component AIDS case reporting systems, but also included HIV surveillance in some population groups $(2,3)$. AIDS case reporting was to become the backbone of surveillance in most industrialized countries, as described in several of the country case studies presented in this supplement. For developing countries, many of which suffer from a dearth of diagnostic facilities, poor health information systems and political and cultural constraints to recording AIDS as a cause of illness or death, AIDS case reporting was to prove woefully inadequate. Instead, many of these countries developed and relied on sentinel systems to detect trends in HIV prevalence in groups with high-risk behavior as well as in the general population. This paper focuses on these sentinel surveillance systems.

We discuss the major purposes of HIV surveillance and describe how the results of surveillance are used in practice. We then provide a very brief overview of the mix of surveillance activities recommended as appropriate for different epidemic situation-epidemics that are concentrated in defined groups with high-risk behavior, 
and epidemics that are well established among heterosexuals in the population at large. The major challenges facing the two types of surveillance systems are how to make best use of behavioral data to inform biological surveillance data. A brief examination of surveillance systems in two developing countries illustrates how these challenges are being met in practice. While there is room for improvement, HIV surveillance systems in many developing countries are relatively robust, and are growing stronger all the time. In most countries, however, insufficient use is made of the information generated by these systems in terms of strengthening HIV prevention and care programs.

\section{PURPOSES OF HIV SURVEILLANCE}

Data generated by HIV surveillance systems can be used in three major ways: to plan HIV prevention and care programs, to monitor the impact of the national response to the epidemic, and to lobby for a more effective response in the future. While these are all broadly compatible, the quality of the data needed for each differs somewhat.

\section{Using Surveillance Data to Inform Programming Decisions}

Providing data that can be used to plan interventions and to inform programming is perhaps the most important function of an HIV surveillance system. HIV surveillance systems allow public health workers to track trends in infection over time, recording the geographical spread of HIV as well as identifying which population groups are infected. Second generation surveillance systems, which incorporate behavioral data collection, are also able to identify populations not yet infected but at risk for infection. The behavioral component of HIV surveillance systems can also contribute information as to which behaviors put people at risk for infection in any given population.

\section{Using Surveillance Data to Judge the Success of the National Response}

Another, and perhaps controversial, use of surveillance data is to monitor and evaluate the national response to HIV. The controversy arises for a number of reasons. First, many argue that flat or falling trends in HIV prevalence can be interpreted in any number of ways. While they may reflect a fall in new infections, they may also reflect a rise in mortality or changes in the population being tested. These issues are discussed at greater length below. A second concern is the extent to which any change in HIV prevalence can be attributed to program activities.

These concerns are valid. Biological surveillance data alone can never be a strong enough basis for making absolute pronouncements about the success or failure of a national HIV prevention program. The addition of behavioral data collection to surveillance systems can suggest reasons for changes in prevalence, but still cannot produce conclusions about causality. Within a larger monitoring and evaluation framework, however, data generated by regular surveillance systems can contribute to an understanding of the impact of the combined effect of the national response to HIV (4-7).

\section{Using Surveillance Data to Lobby for a More Effective Response}

Data generated by surveillance systems can and should be used to advocate for a more effective response to the HIV epidemic, at regional, national, and international levels. Where they exist, these lobbying efforts often concentrate on trying to increase political commitment and total resources available for the response to HIV epidemics that are already well established. Surveillance data can also be used to pinpoint windows of opportunity for early action in populations that display low levels of HIV infection but substantial risk behavior.

\section{Information on Trends Versus Absolute Numbers}

There is a certain amount of tension in surveillance activities between the need to establish trends in risk and patterns of infection and the need to establish absolute numbers (HIV prevalence). For many of the planning, monitoring and advocacy functions mentioned above, age-specific trend data are more than sufficient. For example, biological surveillance systems might establish that HIV infection rates are rising in teenage women, while behavioral surveys show continued early onset of sex and low condom use in premarital partnerships. This information is enough to establish the need for HIV prevention programs that provide appropriate information and services to adolescents. It may be enough, too, to establish that existing HIV prevention efforts in these groups are not achieving their aims. And it can be used to lobby with parents, teachers, and politicians for stronger prevention programs in schools.

In other areas, however, absolute numbers are required. In planning care programs for affected individuals or in planning support programs for their families, for example, governments need to know how many people 
are currently living with HIV. Similarly, any assessment of the impact of HIV on the economy-potentially extremely useful in advocating for a stronger national response to the epidemic - will depend upon a relatively accurate estimate of the number of people likely to sicken and die in a given time frame. Additionally, international organizations often apply strong pressure on countries to produce annual estimates of the absolute number of people living with, and dying from HIV.

Surveillance systems were not initially designed for prevalence estimates and are less well equipped to produce reliable data about the absolute numbers of men, women, and children infected with the virus than they are to provide information about trends. Estimating the number of people living with HIV involves having a clear idea of the size of different populations with different levels of risk for infection, and some idea of the interaction between these populations. This information is rarely readily available.

\section{SURVEILLANCE SYSTEMS FOR DIFFERENT EPIDEMIC STATES}

In the initial years of the HIV pandemic, surveillance systems tended to follow one of two models. The first, based primarily on AIDS case reporting, was common in industrialized countries and in Latin America. The second, based on unlinked anonymous testing of blood from pregnant women, STI (sexually transmitted infection) clinic patients, and occasionally sex workers became the norm in Africa and much of Asia. The use of these at-risk populations in the surveillance systems was based on the assumption that these groups would be the first infected, and ultimately the virus' bridge to the general population.

Over time it has become clear that this progression is not inevitable. The spread of HIV from subpopulations with high risk to any wider population-and its subsequent spread through that wider population-depends largely on patterns of sexual networking between men and women with different levels of risk behavior. Where these patterns of sexual networking do not exist, HIV may well remain concentrated in defined high-risk subpopulations, and surveillance systems that concentrate on testing pregnant women will be inappropriate. In some countries of the former Soviet Union, for example, HIV surveillance systems tested millions of pregnant women and found little in the way of infection, even as the virus raged through injection drug users.

In short, HIV surveillance systems must look for the virus where it is most likely to be found, and must investigate the behavioral links between subpopulations among whom the virus is concentrated and other populations. In epidemics where HIV is already well established in the general population, the focus of surveillance systems will be different. In generalized HIV epidemics, surveillance systems will try to collect information that helps distinguish between the natural evolution of an HIV epidemic and the effects of changing behavior and access to care.

Together with a number of developing countries and international partners, The Joint United Nations Program on HIV/AIDS (UNAIDS) and The World Health Organization (WHO) have developed recommendations for HIV and behavioral surveillance suitable to different epidemic states $(1,8)$. These states are defined in terms of levels and patterns of risk in different populations. For the sake of convenience, numerical proxies are used to distinguish between the epidemic states, as follows:

- Low-level: Although HIV may have been recorded for many years, prevalence has never consistently exceeded 5\% in any subpopulation.

- Concentrated: HIV is well established in subpopulations with known high-risk behavior, such as injection drug users (IDUs), men who have sex with other men (MSM), or sex workers. However, there is no sign of substantial spread beyond these groups. HIV prevalence consistently exceeds $5 \%$ in at least one high-risk group, but remains below $1 \%$ in pregnant women in the general population.

- Generalized: HIV is well established in the general population, with prevalence consistently exceeding $1 \%$ among pregnant women.

In low-level and concentrated HIV epidemics, it is recommended that the bulk of surveillance activity remain concentrated in those subpopulations, which have been demonstrated to engage in behavior that carries a high risk of HIV infection. Biological surveillance should track trends in HIV infection in these groups, while behavioral surveillance should concentrate on understanding the links between these subpopulations and other populations that do not share directly in the highrisk behavior in question.

If HIV infection rises to substantial levels in welldefined subpopulations with high risk, and if behavioral surveillance has shown that these subpopulations are linked to other populations, surveillance activities should be expanded to monitor first behavior and then HIV infection rates in those other populations. Where those other populations are essentially indistinguishable from all sexually active adults, biological and behavioral surveillance in groups representative of the general population should be instituted. 
If HIV is well established in the general population, sentinel surveillance among pregnant women should concentrate on trying to detect changes in infection in women in their teens and early 20s-the groups most likely to reflect recent infections. Behavioral surveillance, too, should pay particular attention to establishing trends in risk behavior among young people. As HIV infection rates in the general population rise, HIV testing for surveillance purposes can be dropped in some groups that may have been useful early in the epidemic, because the data they generate become increasingly difficult to interpret. Patients at STI clinics and blood donors both fall into this category.

In summary, HIV surveillance systems should be appropriate to the current epidemic state, but should be expanded and adapted if and when the existing surveillance system detects changes in the dynamic of the epidemic.

\section{CHALLENGES FOR HIV SURVEILLANCE IN CONCENTRATED EPIDEMICS}

In a concentrated HIV epidemic, the bulk of infections are among people who engage in especially high-risk drug-taking or sexual practices, principally injection drug users, men who have sex with other men, and sex workers and their clients. These groups usually exist on the margins of society, and their activities are often illegal. This makes HIV surveillance extraordinarily difficult, both ethically and practically. Where people are subject to imprisonment or systematic discrimination for their HIV-related behaviors, there must be serious doubts about the wisdom of even trying to identify populations and individuals for HIV surveillance. The validity of selfreported behavioral information gathered from people whose risk behaviors are illegal is also open to question.

\section{Defining Populations at Risk}

A necessary starting point for HIV surveillance activities in concentrated epidemics is to define the populations at risk. A population must be defined before sentinel populations can be selected or sample frames for behavioral surveillance can be constructed. And if estimates of the absolute numbers of people living with HIV are to be made, then some idea is needed of the total size of the at-risk population.

Perhaps the easiest situation for HIV surveillance among high-risk groups is in countries where sex workers are registered, and where they undergo regular screening and treatment of syphilis and other STIs. This provides the HIV surveillance system with easy access to blood samples that can be used in unlinked anonymous testing for HIV. Where the system functions well (such as in Senegal, and the Philippines), it is reasonable to assume that these surveillance results are fairly representative of all registered sex workers. A problem arises where there is a significant population of sex workers who are unregistered or where registered sex workers, when they test positive, continue their work but as unregistered, and now uncounted. This is the case even in most countries where sex work is legal, because of illegal immigration, underage workers, and other factors. These unregistered sex workers may be significantly less likely to be able to negotiate condom use with their clients, and are unlikely to enjoy the same easy access to free STI treatment as their registered peers. HIV prevalence in this group may therefore differ significantly from HIV infection among registered sex workers.

In countries where sex workers are neither brothelbased nor registered, surveillance is harder still. In many countries, even sex work itself is hard to define, with a substantial proportion of women exchanging sex with a number of men for money, support, or favors, without considering themselves sex workers, and without being considered sex workers by their patrons. In terms of HIV surveillance, the issue is not how women or their clients perceive themselves, but whether their behavior contributes to an elevated risk of HIV transmission because of partner turnover or condom use patterns.

Men who have sex with other men are another population that can be hard to define. In some countries, a well-established gay scene provides easy access to this group. But the very use of the phrase "men who have sex with men" rather than gay or homosexual men suggests that elsewhere men may fall into this risk category without considering themselves to be members of a defined subpopulation. This appears to be the case particularly in parts of Asia and Latin America (9). It is certainly difficult to establish any kind of representative sampling process for either behavioral or biological surveillance within a high-risk group if members of that group do not consider themselves to belong to it. Estimating total size of the population engaged in male-male sex is also very difficult outside of an established gay scene.

Injecting drug users are easier to define, but harder still to count. Drug injection is probably the most universally marginalized of all HIV-related behaviors, and it is also almost universally illegal. Recent estimates of the number of injection drug users in Russia, for example, vary by a factor of 10 (10).

\section{Accessing Populations with High-Risk Behavior}

In order to carry out effective sentinel surveillance for any disease, it is necessary not just to define a popula- 
tion, but also to find a sentinel population that is in some way representative of that group. Defining the relationship between the sentinel population and the larger population of people to whom results of sentinel surveillance may be extrapolated is an enormous challenge in concentrated HIV epidemics.

A major difference is between sentinel surveillance that is based in a service provision setting (e.g., prenatal or STI clinics) and those that must be linked to some newly established services or intervention. In the routine, clinic-based surveillance setting it is easier to obtain a more representative sample of the populations being tested, and the testing can be done with samples drawn for other purposes. Surveillance of many high-risk groups is not so easy.

As mentioned, sex workers who are regularly screened and treated for STIs may provide an ideal sentinel population for HIV surveillance in some concentrated epidemics. But those results cannot reliably be extrapolated to unregistered sex workers. Recruiting MSM for voluntary unlinked anonymous testing is possible where there is a defined gay scene, although men recruited at common gay gathering sites such as bars or bathhouses may not be typical of the entire gay population. HIV surveillance among drug injectors has most commonly been associated with either treatment programs or imprisonment-neither likely to produce results representative of all drug injectors.

Ethically, most biological as well as behavioral surveillance among high-risk groups should be associated with HIV prevention programs and other service provision among the marginalized groups in question. As well as producing results that are not representative of all people sharing the risk behavior of interest, this will probably produce changing biases over time.

\section{CHALLENGES FOR HIV SURVEILLANCE IN GENERALIZED EPIDEMICS}

The challenges posed by sentinel-surveillance based systems in generalized epidemics are very different from those encountered in concentrated epidemics. Generally, there is little difficulty in defining the at-risk population-all sexually active adults. Unlinked anonymous testing of pregnant women attending (sentinel) prenatal clinics has proved a feasible and affordable alternative to population-based studies, and has in many countries provided consistent and credible trend data over time. As the HIV epidemic matures in the badly affected countries of sub-Saharan Africa, it is becoming apparent that there are still significant challenges associated with surveillance.

\section{The Relationship Between Tested Pregnant Women and All Women}

Pregnant women were originally selected as a sentinel population for HIV surveillance as a way to monitor trends in a population group at low risk of infection. This population was selected because the coverage by prenatal clinics was high in most developing countries and because blood was already being drawn for other purposes $(1,11)$. Indeed, soon after it became apparent that unprotected sex between men and women was the force driving prevalence in generalized epidemics, HIV prevalence data measured in pregnant women aged 15-49 were commonly applied to the whole adult population, male and female, sexually active and otherwise, to derive estimates of the number of adults living with HIV.

Using this group as the basis of estimates for the general adult population has limitations. Recent work on fertility and studies comparing infection rates recorded in prenatal clinics to prevalence in household studies reveals that this assumption, while broadly valid, should be treated with some caution (12).

Older HIV-infected women are unlikely to become pregnant as HIV infection acts to depress fertility progressively over the course of a person's infected life. Because of this, these women are less likely to be included in the tested population. At younger ages, however, only a fraction of the population is sexually active, so a substantial proportion may not be exposed to any risk either of pregnancy or of HIV infection. These factors mean that in mature, generalized HIV epidemics, prenatal clinic data tend to overestimate HIV prevalence in the youngest age groups and underestimate it in older age groups compared with women in the general population. Because the fertility effect is already apparent in women in their mid-20s, the net result across the 15-to49-year age range is that prenatal clinic data tend to underestimate HIV infection among all adult women.

The assumption that pregnant women represent all sexually active women becomes more tenuous as use of modern contraceptives becomes more common. In many countries where HIV prevalence is high, contraceptive use has also risen significantly in recent years. Over half of all women aged 15-49 were using contraception in South Africa in 1998, with 39\% of Kenyans using contraception the same year. Some $48 \%$ of women in Zimbabwe were using contraception as early as 1994. At least in some countries, contraceptive use continues to rise. In Kenya for example, use of modern contraceptive methods more than tripled from $10 \%$ in 1984 to $32 \%$ in 1998 (13). In all of these populations, the use of condoms as a contraceptive method was low. Women who use 
non-barrier contraceptives are exposed to the risk of HIV infection without being exposed to the risk of pregnancy. Changes in contraceptive use over time may therefore affect trends over time in the relationship between HIV prevalence recorded in surveillance at prenatal sites and real levels of infection in the female population.

Another potential bias in HIV prevalence data derived from sentinel surveillance among women at prenatal clinics arises as a result of site selection. In most countries, sentinel sites are confined to public prenatal clinics. Coverage of public prenatal clinics is therefore an important factor, particularly if women attending these clinics come from selected groups (geographic, socioeconomic, etc.) As health systems are restructured and economic conditions change, the profile of women attending government clinics may also change. If this change is associated with socio-cultural or economic factors that are also related to the risk of being HIVinfected, trends in HIV prevalence over time may be affected. It has been shown that changes in the population attending prenatal clinics where surveillance is conducted can have a significant impact on outcome of surveillance (14).

\section{HIV Surveillance in Men in Generalized HIV Epidemics}

For all the limitations discussed above, pregnant women attending prenatal clinics provide a convenient sentinel population that poses few ethical problems and relates in fairly predictable ways to a larger population. In most countries, no such population is available for men.

In most cases, surveillance systems in generalized epidemics have simply taken HIV rates recorded in pregnant women and applied them to men aged 15-49. A growing number of population-based studies now suggest that this is not a valid approach. They suggest, that HIV prevalence has become higher among women aged 15-49 than among men in the same age range in many countries in sub-Saharan Africa. Women are generally infected younger than men in the countries worst affected by HIV, so part of this is a demographic effect. However there is also evidence that people infected at younger ages survive longer with HIV (15). This, too, contributes to higher HIV prevalence among women than men in generalized epidemics, assuming that HIV incidence rates across the whole 15-49 year age range are broadly similar between the sexes.

Many countries have used data from blood donors and of men presenting at STI clinics to provide some surveillance data on men considered to be at low risk and at high risk of HIV infection, respectively. Most countries now have prescreening procedures for blood donors, thus excluding those who self-report a sexual history that might expose them to higher than average risk of HIV. Trend data from STI patients have also become very difficult to interpret, since men responding to HIV prevention campaigns by cutting out risky sex will drop out of the numerator, leaving only those with the highest risk behavior in the tested population. HIV prevalence rates among STI clinic patients may therefore continue to rise even in the presence of overall trends toward safer behavior.

A handful of countries have easily accessible male sentinel populations such as new military recruits, although these rarely cover the whole at-risk age range. One alternative is to conduct occasional populationbased surveys among men and women that include unlinked anonymous testing for HIV. These can be biased by high refusal and non-response rates, especially among men who are frequent travelers (and may therefore also have higher than average levels of risk behavior). The experience of some 10 sub-Saharan African countries suggests, however, that such surveys can provide information that is invaluable in both assessing the reliability of regular surveillance systems, and making adjustments so that data gathered among pregnant women can plausibly be extrapolated to the male population.

\section{Interpreting Trends in Surveillance Data in Generalized HIV Epidemics}

In the early years of generalized epidemics, the trend in prevalence tends to be upwards at all ages. This presents few difficulties in interpretation. The picture is complicated when prevalence begins to flatten or fall. Are the changes the result of fewer new infections, of rising mortality or of changes in the tested population? A single figure for prevalence in persons aged 15-49 years cannot answer these questions.

Second generation surveillance systems attempt to increase a system's ability to explain observed changes in two major ways. The first is by disaggregating data by age and looking more closely at the younger age groups, where fertility and mortality biases are less pronounced and where prevalence more nearly approximates incidence. The second is by supplementing biological data with behavioral data, to investigate whether there are any changes in the proximate determinants of HIV infection that might plausibly explain observed changes in prevalence among young people. 


\section{The Role of Repeated Behavioral Surveys in HIV Surveillance}

The role of repeated behavioral data collection differs in concentrated and in generalized epidemics. In low level and concentrated epidemics, behavioral data can be used as an effective early warning system, signaling the presence of high-risk behaviors even before HIV infection rises to measurable levels in the at-risk population. Behavioral data can also highlight sexual links between subpopulations with especially high risk of HIV infection and other, broader populations. This can identify population groups that should be included in future rounds of surveillance. Finally, behavioral surveillance in subpopulations with high-risk behavior can be used to monitor the progress of the sum of interventions aimed at establishing safer behaviors within those populations.

In generalized epidemics the major role of repeated behavioral data collection is, as suggested above, to help explain observed changes in HIV prevalence. If lower HIV infection and STI rates are preceded by a fall in risky sexual partnerships and a rise in condom use, it is entirely plausible to assume that the biological data reflect a fall in new infections (16). If, on the other hand, a flattening in prevalence occurs in the absence of any apparent decrease in risky sex, a rise in death masking continued high HIV incidence cannot plausibly be ruled out.

In both cases, behavioral data can contribute significantly to the advocacy value of the HIV surveillance system. This may be especially true in countries such as Senegal, where concerted prevention efforts have contributed to keeping HIV infection rates in the general population low. Flat prevalence, even at low levels, does not impress policy makers and donors as much as falling prevalence of the sort measured by the biological surveillance system in Uganda (4). Accompanied by behavioral data showing rises in condom use in risky partnerships and rising age at first sex, however, the biological data provides evidence of the success of prevention efforts even in relatively low-prevalence HIV epidemics.

Behavioral data is also important in achieving the first function of HIV surveillance - to inform programming that aims to reduce the continued spread of the virus. Regular monitoring of HIV-related behavior can reveal which risk behaviors persist despite prevention efforts, and suggest where renewed program efforts are needed.

Behavioral data collection is not without its shortcomings. Identifying at-risk populations and sampling them in a way that gives reliable trends over time consumes time and resources, especially in marginalized groups. While ethical concerns are less pronounced in behavioral surveys than in biological surveys, they remain significant where risk behavior is illegal or may lead to discrimination.

In addition, there are persistent concerns about the validity of self-reported information about socially unacceptable behaviors. While these concerns are valid to an extent, years of experience with fertility surveys and other surveys of sexual behavior suggest quite good agreement between self-reported behavior and other indicators such as STI prevalence and condom sales figures. It appears that many of the sources of bias in behavioral data affect the reporting of absolute levels of risk behavior more than they affect trends over time. For the purposes of HIV surveillance, therefore, repeated behavioral surveys could yield quite reliable information. (8)

A further methodologic challenge for behavioral surveillance concerns the definition of risky sexual partnerships. Clearly, the higher the background prevalence of HIV, the greater the risk inherent in having unprotected sex with any partner, including "regular" or marital partners. Methodologic work is continuing to try to define indicators that better capture the risk inherent in different patterns of sexual networking in high prevalence HIV epidemics.

\section{SENTINEL HIV SURVEILLANCE IN PRACTICE: HOW CLOSE ARE WE TO THE SECOND GENERATION?}

The outlined challenges have only recently been clearly defined, and many of the more difficult issues have yet to be resolved. WHO, UNAIDS and their partners are currently engaged in an initiative to support a number of countries in strengthening their existing surveillance systems and in integrating second generation surveillance more closely into a wider monitoring and evaluation framework. Certainly, there is much to be done. The weakest area at present remains surveillance in subpopulations with high-risk behavior in concentrated epidemics. Behavioral surveillance systems in generalized epidemics are also inadequate, with no more than a handful of countries having established systems to ensure the repeated monitoring of risk behaviors.

Nevertheless, many countries are working hard to improve their existing surveillance systems, and there are several examples of good practice to be found even in countries with very limited resources.

Botswana is an example of a country with a generalized epidemic that has instituted and maintained a strong sentinel surveillance system. Since 1990, Botswana has collected data from prenatal clinics located in both urban 
and rural areas. Data were also collected from STI clinics. A core set of prenatal clinic sites has been used over time so that prevalence trends may be monitored unconfounded by the possibility of differences due to geographic location. New prenatal clinic sites have been added each year, as the epidemic has grown. In 1998, Benin had three sites located in urban areas and six sites in rural areas. For a small country with a population of less than 1.6 million people, this surveillance system provides high quality information that can be used to judge the current level of the epidemic and provide information about its spread into new geographic regions in the country. Also, as data are reported by age groups, estimates of the trend in new infections can be made. Overall, Botswana's surveillance system is very effective.

Vietnam is a country with a low-level or concentrated epidemic and has, since the first reported HIV case in 1990, established an extensive sentinel surveillance system. In 1991 the first round of surveillance was performed among prenatal clinic attendees, commercial sex workers, and IDUs in Ho Chi Minh City. Broad surveillance activities began in 1994, with sentinel sites in four provinces. This was expanded to sites in twenty provinces in 1996. The high-risk populations tested are commercial sex workers, military recruits, IDUs, and attendees at TB and STI clinics. In addition, there are sentinel surveillance sites at prenatal clinics in twenty provinces. In addition to the sentinel surveillance sites, there is mandatory HIV and AIDS case reporting in the country.

One of the problems that face countries like Vietnam is in obtaining representative samples for high-risk populations. The samples used for these populations are comprised of those people who have been arrested by the police or have been caught up in a sweep by the authorities. While such samples as these do provide useful data, they are not representative samples and do little to promote a sense of trust between the members of these populations and authorities. This trust is necessary to implement the harm reduction programs that are so important in reducing the spread of HIV in these same populations.

\section{The Quality of HIV Surveillance Data in Developing Countries}

HIV surveillance systems in developing countries are far from perfect. Biases exist in the data, especially in concentrated epidemics, although the direction of these biases is often predictable. It is worth reflecting on the quality of HIV surveillance data and estimates of HIV infection as compared with other diseases.
First, many countries have established sentinel surveillance systems that have measured HIV prevalence in the same or similar populations in different sites for several years. Analysis of population-based HIV prevalence studies have allowed for a fair comparison between sentinel surveillance and general population prevalence in a number of settings, and sentinel surveillance has generally measured up exceptionally well.

There is a significant gap in data collection in some populations, notably in the truly rural areas that are home to a significant proportion of the population in the badly affected countries of sub-Saharan Africa. Existing estimates for rural populations are often based on sentinel sites in peri-urban areas or regional trading hubs, which may not be representative of rural areas. Despite these shortcomings, HIV surveillance systems provide measures of HIV prevalence in thousands of sites around the developing world on a regular basis.

The same cannot be said of many other diseases. A recent review of tuberculosis estimates in sub-Saharan Africa, for example, revealed that TB prevalence studies in the general population were only available for two countries (17). One dated from 1989, and both were rated as having poor data quality. The study's authors state that "Estimates (of the global prevalence of TB) derived from relatively poor data were strongly influenced by panel member opinion." It is a tribute to HIV surveillance systems in developing countries that this is not the case for HIV. A few populous countries such as India and Nigeria have not yet built up reliable time series in HIV prevalence data, and current estimates of infection in those countries are liable to change considerably, as surveillance systems improve. In general, however, HIV surveillance systems in developing countries, and especially in sub-Saharan Africa, produce regular, timely and reliable data. Efforts to strengthen existing surveillance systems can only improve this situation.

\section{CONCLUSION}

Existing HIV surveillance systems in developing countries face continuing challenges, particularly in countries with concentrated epidemics (18). Improving methodologies and the expansion of behavioral surveillance systems will help meet some of these challenges.

Overall, the quality of data generated by existing HIV surveillance systems is good, particularly in countries with generalized epidemics. Indeed the quality of data generated by HIV surveillance systems exceeds that generated by surveillance systems for most other diseases in developing countries. 
A major challenge for the developing world is not only to generate better data on HIV prevalence but also to make better use of the data that are currently produced. The failure of a large number of countries to turn good information about HIV and the behaviors that spread it into effective programs to reduce its spread and properly care for those infected is one of the abiding weaknesses of the international response to HIV.

\section{REFERENCES}

1. UNAIDS/WHO. Second generation surveillance for HIV/AIDS: the next decade. Geneva: UNAIDS, 1999.

2. Chin J. Public health surveillance of AIDS and HIV infections, Bull World Health Org 1990;68:529-36.

3. WHO/GPA. Report of a meeting on HIV sentinel surveillance, Dakar, Senegal. December 14-18, 1991. (GPA/CNP/EVA/92.2)

4. Asiimwe-Okiror G, Oppio A, Musinguzi J, et al. Change in sexual behavior and decline in HIV infection among young pregnant women in urban Uganda. AIDS 1997;14:1757-63.

5. Meda N, Ndoye I, M'Boup S, et al. Low and stable HIV infection rates in Senegal: natural course of the epidemic or evidence for success of prevention? AIDS 1999;13:1397-1405.

6. UNAIDS. Relationships of HIV and STD declines in Thailand to behavioral change. UNAIDS, Geneva 1998.

7. UNAIDS/MEASURE Evaluation. National AIDS programs: a guide for monitoring and evaluation. Geneva. UNAIDS 2000

8. Family Health International, IMPACT, UNAIDS. Meeting the behavioral data collection needs of national HIV/AIDS and STD programs. FHI, Washington DC, 1998.
9. Kerr-Pontes LRS, Gondim R, Mota R, et al. Self-reported behavior and HIV risk taking among men who have sex with men in Fortaleza, Brazil. AIDS 1999;13:709-17.

10. Dehne KL. The HIV epidemic in Central and Eastern Europe: update. Paper presented at UNAIDS and UNICEF sponsored Strategy Meeting to Better Coordinate Regional Support for National Responses to HIV/AIDS in Eastern and Central Europe. Geneva, October 1999.

11. Unlinked anonymous screening for the public health surveillance of HIV infections: proposed international guidelines. WHO document GPA/SF1/89.3. Geneva, 1989.

12. Zaba B, Gregson S. Measuring the impact of HIV on fertility in Africa. AIDS 1998;12(Suppl 1): S41-50.

13. Kenya, National Council for Population and Development. Kenya Demographic and Health Survey 1998. Macro International, Calverton Maryland 1999.

14. Jackson D, Ngugi E, Plummer F, et al. Stable antenatal HIV-1 seroprevalence with high population mobility and marked seroprevalence variation among sentinel sites within Nairobi, Kenya. AIDS 1999;13:583-9.

15. Darby S, Ewart D, Giangrande P, et al. Importance of age at infection with HIV-1 survival and development of AIDS in UK haemophilia population. Lancet 1996;347:1573-9.

16. Nelson K, Celentano D, Eiumtrakol S, et al. Changes in sexual behavior and a decline in HIV infection among young men in Thailand. N Engl J Med 1996;335:297-303.

17. Dye C, Scheele S, Dolin P, et al. Global burden of tuberculosis: estimated incidence, prevalence and mortality by country. JAMA 1999;282:677-86.

18. Walker N, Garcia-Calleja JM, Heaton L, et al. Epidemiological analysis of the quality of HIV sero-surveillance in the world: how well do we track the epidemic? AIDS 2001;15:1545-54. 